\title{
A rapid improvement program. How to standardise a joint replacement pathway across 10 hospitals in 6 months.
}

\author{
Tom Wainwright and Robert Middleton, Directors of Healthdecoded Limited. \\ Mark Ferreira, Clinical Implementation Manager of General Healthcare Group.
}

International Forum on Quality and Safety in Health Care, 5-8 April 20II, Amsterdam.

\section{Introduction}

There is strong evidence for implementing standardised enhanced recovery pathways after hip and knee replacement surgery. However, many units still do not use defined clinical pathways. A project was therefore undertaken to introduce a standard clinical pathway across a group of private hospitals.

\section{Background}

BMI Healthcare identified that there was significant variation across their 60 hospitals in regard to length of stay following hip and knee replacement. Diagnostic investigations into this variation revealed significant variation in the care processes and procedures used at individual hospitals and across units. Therefore, ten hospitals were chosen as sentinel sites, and a standardised pathway was introduced. In each hospital the entire multi- disciplinary team was involved in the project.

\section{Intervention}

A 6-month rapid improvement program was undertaken. The first step identified best practice for the provision of hip and knee replacement pathways. An evaluation of current clinical pathways against the gold standard established from the scoping exercise was then completed in each unit.

A central 'improvement team' in each hospital were trained in quality improvement techniques and they facilitated the changes in each hospital. The pathway was introduced along with improved documentation and performance measures. This was followed by an evaluation of the new pathway.

Regular monitoring was completed centrally via email and telephone, as well as monthly site visits where the hospital team presented their progress, and difficulties with implementation were addressed.

\section{Effects of changes}

The new pathway was successfully implemented by all 10 hospitals. This is evidenced by full compliance to the new pathway, which was measured in each unit using variance analysis and process control measures. The new pathway resulted in a 14\% reduction to length of hospital stay across the nine sites, excellent clinical outcomes, and high patient and staff satisfaction.

\section{Message for others}

Facilitating change in ten separate hospitals concurrently is possible if sound quality improvement methodology is used, along with regular monitoring, and the involvement of the whole MDT team in each hospital.

The role of ongoing monitoring from external experts and monthly performance reports for each hospital was a very powerful method for making change happen.

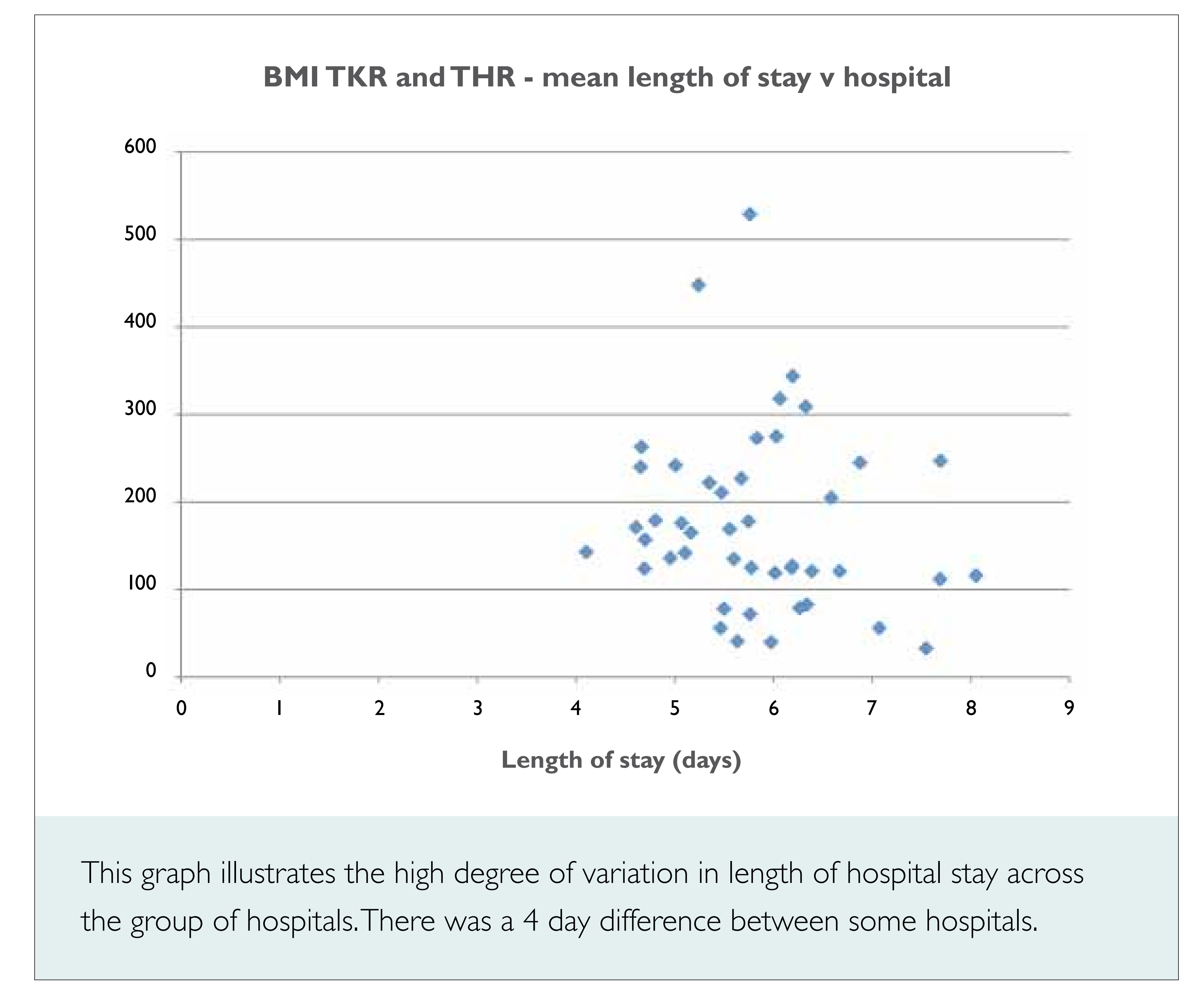

BMI TKR and THR - Reduction of LOS in 10 sentinel sites after 6 months

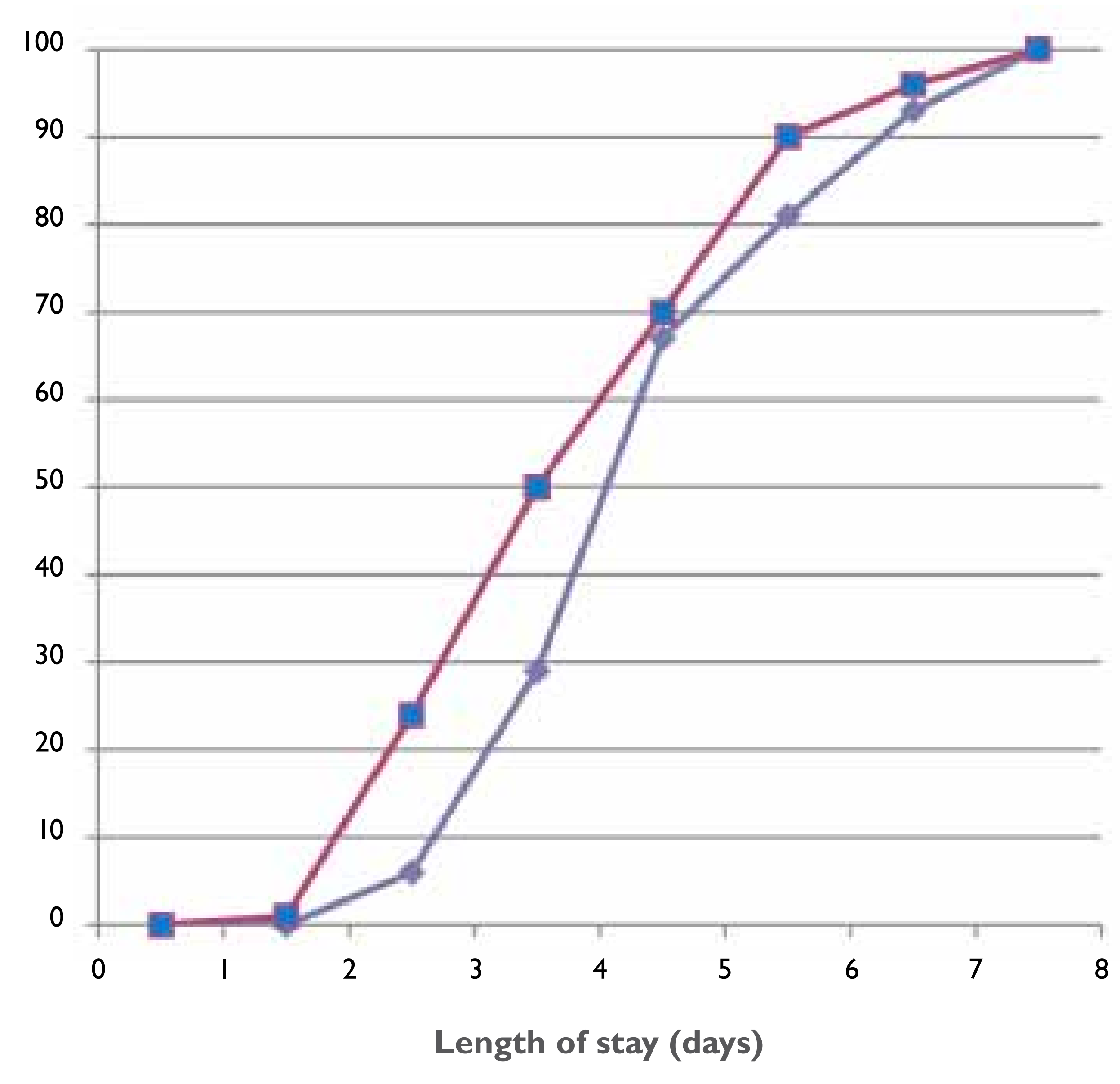

This graph illustrates the change in length of stay of the 10 seutinel sites that were part of the project. The pink line indicates LOS after the project. There was an overall $15 \%$ reduction in LOS and a marked increase to the number of discharges on the third and fourth post-operative days. 